\title{
Recursos Educacionais Abertos: Uma Análise dos Objetivos de Aprendizagem Referenciados pela Taxonomia Digital de Bloom
}

\author{
José Oxlei de Souza Ortiz ${ }^{1}$, Viviani Kwecko², Fernando Tolêdo ${ }^{3}$, Sam Devincenzi ${ }^{4}$, \\ Silvia Silva da Costa Botelho 5
}

1,2,3,5 Universidade Federal do Rio Grande (FURG) - Rio Grande - RS - Brasil

${ }^{4}$ Universidade Federal do Pampa - Campus Alegrete - RS - Brasil

joxlei@hotmail.com, viviani.kwecko@riogrande.ifrs.edu.br, fernando_ecomeyahoo.com.br, sam.devincenzi@gmail.com, silviacb.botelho@gmail.com

\begin{abstract}
This article presents an analysis of educational metadata related to the learning objectives employed in Educational Objects and in teachers' activities at PhET Colorado. We have as main question, how the teachers have appropriated the educational objects available in PhET for the construction of activities. To this end, the research is based on the cognitive principles of Bloom's digital taxonomy and its adaptations to classify learning objectives. As a result, we found that teachers apply learning objectives in a more diversified way in the construction of their activities, and explore the six levels of taxonomy in a more harmonious way.
\end{abstract}

Resumo. Este artigo apresenta uma análise dos metadados educacionais relacionados aos objetivos de aprendizagem empregados nos Objetos Educacionais e em atividades dos professores no PhET Colorado. Temos como questionamento principal, como os professores têm se apropriado dos objetos educacionais disponíveis no PhET para a construção de atividades. Para tanto, a pesquisa se apoia nos princípios cognitivos da taxonomia digital de Bloom e suas adaptações para classificar os objetivos de aprendizagem. Como resultado verificamos que os professores aplicam de modo mais diversificado os objetivos de aprendizagem na construção de suas atividades, e exploram de modo mais harmônico os seis níveis da taxonomia.

\section{Introdução}

No atual panorama mundial movido pelo conhecimento, os sistemas de ensino tem enfrentado um grande desafio na produção de material educacional para o meio digital. Frente a isso, surgem a utilização de Recursos Educacionais Abertos (REA) como uma potencialidade pedagógica para uso coletivo. Os REA "são materiais de suporte à educação que podem ser acessados, utilizados, modificados e compartilhados livremente" [UNESCO 2015 p.1]. Nas últimas duas décadas, uma quantidade crescente de REA tornou-se disponível para que os educadores utilizem, reutilizem, re-publiquem e compartilhem o conhecimento apoiando o aprendizado colaborativo [Clements, Pawlowski \& Manouselis 2015]. 
Atualmente existe na internet um grande número de repositórios cujo objetivo é armazenar, classificar e disponibilizar os objetos educacionais [Diana 2015]. Como exemplos temos o $\mathrm{MIT}^{1}$ Opencoursewar, que disponibiliza vídeos, livros, textos e aulas organizadas por área de conhecimento, o MERLOT ${ }^{2}$, disponibiliza coleções de materiais didático-pedagógicos e ferramentas de criação de conteúdo, e temos também o PhET $\mathrm{COLORADO}^{3}$, que oferece mais de 150 simulações interativas e 2000 atividades enviadas por professores, que abrangem tópicos em física, química, biologia, ciências da terra e matemática. Trazemos o PhET como destaque devido ao grande fluxo de acesso aos objetos educacionais, com mais de 750 milhões de simulações acessadas.

A utilização desses recursos promove uma cultura de educação aberta e de constante atualização, estabelecendo assim uma importante relação com o contexto atual de educação digital [Mattar, 2013]. Com isso, o uso dos REA vem contribuir no desenvolvimento e melhoria dos materiais educacionais e dos currículos, auxiliando no desenvolvimento do ensino e aprendizagem de qualidade. Sendo assim, o potencial transformador dos REA inclui também a colaboração e compartilhamento, potencializando o papel da criatividade e inovação dos REA na criação de novos modelos educacionais [UNESCO 2015].

Muitos dos recursos educacionais trazem na estrutura de seus Objetos Educacionais (OE) o uso de planos de aulas, exercícios e atividades relacionadas aos conteúdos. Esses materiais abrigam, na grande maioria, informações a respeito do objeto e seu possível uso, bem como informações pedagógicas e objetivos de aprendizagem. No processo de ensino e aprendizagem, decidir e definir os objetivos a serem alcançados passa por articular o fazer educacional para que este possibilite o estabelecimento de novas estruturas cognitivas no educando. Portanto, os objetivos estão no centro do processo de planejamento, defini-los significa delinear o processo de aprendizagem, bem como, torná-lo mais fácil, agradável e significativo [Gil, 2006].

Portanto, este artigo analisa os diferentes objetos educacionais registrados na base de dados do PhET Colorado, com objetivo de identificar os objetivos de aprendizagem que encontram-se dispostos nos objetos educacionais e nas atividades produzidas pelos professores. Tendo como questionamento principal: como os professores têm se apropriado dos objetos educacionais disponíveis no PhET para a construção de atividades? Observando principalmente os objetivos de aprendizagem que os mesmos utilizam na descrição de suas atividades.

O artigo está organizado da seguinte forma. A Seção 2 apresenta o domínio cognitivo da taxonomia digital de Bloom, na Seção 3 temos o processo metodológico com a utilização da taxonomia, para traçar um modelo de análise das características descritas nos metadados educacionais de cada objeto da base citada, a Seção 4 são apresentados os resultados e discussões na análise do REA e, finalmente, na Seção 5 temos as conclusões do trabalho.

\footnotetext{
${ }^{1}$ MIT Opencourseware - https://ocw.mit.edu/index.htm [Acessado: 31-Jan-2020].

${ }^{2}$ MERLOT - https://www.merlot.org/merlot/index.htm [Acessado: 31-Jan-2020].

${ }^{3}$ PhET COLORADO - https://phet.colorado.edu/ [Acessado: 20-Jan-2020].
} 
IX Congresso Brasileiro de Informática na Educação (CBIE 2020)

Anais do XXXI Simpósio Brasileiro de Informática na Educação (SBIE 2020)

\section{Domínio cognitivo da taxonomia de Bloom}

A Taxonomia de Bloom [Lomena, 2006; Guskey, 2001; Bloom et al.,1956; Bloom, 1972; School of Education, 2005; Clark, 2006] foi estruturada a partir de um conjunto de teorias instrucionais com o objetivo de oferecer estratégias diferenciadas para facilitar e avaliar o desempenho de estudantes em diferentes níveis de aquisição de conhecimento, bem como auxiliar os educadores durante o processo educacional de forma estruturada e consciente. Originalmente a taxonomia de Bloom foi estruturada a partir de três domínios: (i)Cognitivo, (ii)Afetivo e (iii)Psicomotor.

Em 2001 a Taxonomia de Bloom foi revisada por Krathwohl e Anderson (2001) que concederam maior ênfase à efetividade do processo educacional, ampliando o alcance desse planejamento para além da atividade em si. Essa revisão da taxonomia propôs uma reorganização, principalmente nos itens superiores da taxonomia. Para o nível cognitivo, o autor estabelece um conjunto de seis categorias para orientar o planejamento educacional: Recordar, Entender, Aplicar, Analisar, Avaliar e Criar. O nível mais básico é o "Recordar", por exigir dos alunos a retomada de conceitos já apreendidos, enquanto que o nível mais complexo é o "Criar", que exige a combinação de diferentes fontes de conhecimento [Dorodchi, Dehbozorgi \& Frevert, 2017]. Assim a taxonomia é um modo de classificar os níveis de aprendizado para formatar resultados instrucionais mensuráveis.

Em 2009 a taxonomia de Bloom revisada foi adaptada por Churches (2009) para abordagem de recursos educacionais em ambientes de aprendizagem digital, conforme Figura 1. Os objetivos, processos e ações oriundas das Tecnologias da Informação e Comunicação (TIC's), passaram a exigir a implementação de novas camadas (verbos secundários) àqueles abordados na taxonomia. Essa adequação, justificada pelo aumento de aprendizagens em contexto tecnológico apresenta uma complementação aos verbos principais, detalhando uma segunda camada de verbos secundários que ajudam na aplicação dessa abordagem metodológica. A partir desse conjunto de etapas de sistema de planejamento são evidenciadas as habilidades de pensamento que partem desde o nível inferior até a habilidades de ordem superior.

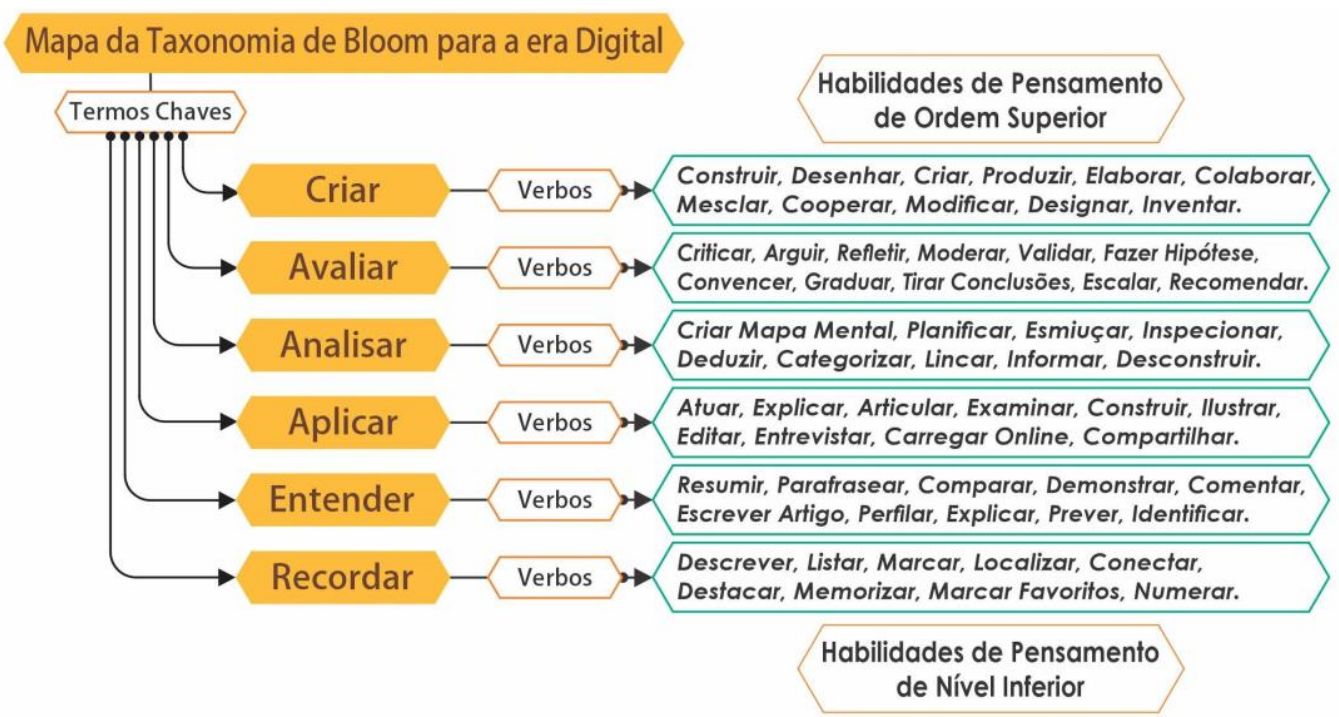

Figura 1. Taxonomia de Bloom para era digital 
Neste sentido, a taxonomia Bloom para era digital apresenta uma organização cognitiva, por propor um conjunto de categorias com a utilização de uma variedade de verbos que buscam orientar o planejamento educacional para o contexto digital. Esses verbos promovem uma série de ações cognitivas dentro das categorias principais, sendo construído com base nas lembranças e compreensões dos conhecimentos para levar os estudantes a usar e aplicar habilidades, envolvendo análise e avaliação de processos, consequências e resultados para que eles elaborem, criem e inovem [Churches, 2009].

\section{Processo metodológico}

A pesquisa realizou uma análise dos objetivos de aprendizagem utilizados na descrição dos objetos educacionais disposto no PhET Colorado. Buscou-se analisar nos objetivos de aprendizagem de cada material a presença de diferentes níveis de desenvolvimento cognitivo, para isto, utilizamos a taxonomia de Bloom para era digital elaborado por Churches (2009) e referenciada nas pesquisas de Dorodchi, Dehbozorgi \& Frevert, (2017) e Wiley (2002) ao modo de classificá-los.

O PhET disponibiliza mais de 150 simulações interativas denominadas de Objetos Educacionais $(\mathrm{OE})$. Esses objetos abrangem vários tópicos nas áreas da física, química, biologia, matemática e ciências da terra, com mais 2000 atividades relacionadas traduzidas para mais de 90 idiomas. O PhET disponibiliza os $\mathrm{OE}$ e atividades em níveis de conhecimento de aprendizagem, sendo elas: Escola primária, Ensino fundamental, Ensino Médio e Universidade. O recurso educacional PhET tem como base de funcionamento fornecer $\mathrm{OE}$ e juntamente disponibilizar uma série de atividades relacionadas que são postadas por professores de várias partes do mundo. Referente a quantidade de atividades que cada OE possui, esse número varia muito, sendo que alguns contém um número pouco expressivo de atividades, enquanto outros fornecem um número superior a 50 atividades relacionadas.

Como recorte desta pesquisa escolhemos a categoria associada ao Ensino Médio que abrange as cinco áreas do conhecimento. Na categoria Ensino Médio temos mais $120 \mathrm{OE}$ originais do PhET e suas respectivas atividades. Desse total de OE, foram retiradas de forma aleatória uma amostra de $20 \mathrm{OE}$ e desses foram retirados 05 atividades de cada, totalizando 100 atividades dos professores. Para a análise dos objetivos de aprendizagem dos $\mathrm{OE}$ do $\mathrm{PhET}$, propõe-se duas etapas metodológicas: (i) coleta e classificação dos objetivos de aprendizagem dos $\mathrm{OE}$ do PhET original, sendo esses objetivos elaborados pela própria equipe de design do recurso; (ii) coleta e classificação dos objetivos de aprendizagem das atividades que foram postadas pelos professores que utilizam os $\mathrm{OE}$ via plataforma PhET. Ambas análises foram referenciadas pela taxonomia digital de Bloom, para formar um material de subsídio para as discussões.

Referente a primeira etapa observamos que o PhET adota um design personalizado para disponibilizar as informações em seus objetos educacionais ao público na web. Cada OE/simulação tem seis campos de metadados contendo as seguintes informações: ABOUT (informações pedagógicas do objeto), FOR TEACHERS (informações para os professores), TRANSLATIONS (idiomas disponíveis), RELATED SIMULATIONS (simulações relacionadas), SOFTWARE 
REQUIREMENTS (requisitos técnicos para funcionamento) e CREDITS (Equipe de Design, bibliotecas de terceiros e indicação de autores).

A partir do conjunto de informações presente no metadado optamos em analisar o campo Sample Learning Goals (objetivos de aprendizagem) vinculado ao ABOUT, esse campo indica os objetivos de aprendizagem que o recurso se dispõem a realizar durante o uso. Esses objetivos de aprendizagem tem uma função principal de auxiliar os professores nas atividades. Dos 20 OE selecionados e seus respectivos objetivos de aprendizagem, obtivemos um total de 94 itens, que foram analisados e classificados com suas indicações de níveis cognitivos. Assim, obtivemos um percentual de uso cognitivo nos objetivos para cada nível da taxonomia, que podemos verificar nos resultados da pesquisa.

Para a segunda etapa da análise, utilizamos os mesmos 20 OE para extrair as 05 atividades propostas por professores, nas quais mapeamos os objetivos de aprendizagem de cada uma, para análise e classificação utilizamos o mesmo processo anterior. Dessas 100 atividades obtivemos um total de 326 objetivos de aprendizagem.

O processo de classificação dos objetivos de aprendizagem, utiliza os níveis cognitivos da taxonomia digital de Bloom elaborado por Churches (2009). Com isso, para cada objetivo de aprendizagem encontrado atribuímos um nível cognitivo da taxonomia, relacionando a intencionalidade proposta no objetivo com as ações de cada verbo da taxonomia. Como exemplo, na Tabela 1 é apresentada a extração dos objetivos de aprendizagem de $1 \mathrm{OE}$ PhET original (simulação - adição de vetores), no qual apontam que permeia os níveis recordar, entender, aplicar e analisar. Assim, podemos observar no primeiro objetivo, "Descrever um vetor com suas próprias palavras" a intencionalidade em descrever, essa ação se relaciona com o nível de recordar conforme a classificação dos verbos elaborado por Churches (2009).

Tabela 1. Análise do campo objetivos de aprendizagem do OE adição de vetores

\begin{tabular}{|l|c|}
\hline \multicolumn{1}{|c|}{ Objetivos de Aprendizagem ( OE Phet originais ) } & Verb. Taxonomia \\
\hline Descreve um vetor com suas próprias palavras & Recordar \\
\hline Explicar um método para adicionar vetores & Entender \\
\hline Comparar e contrastar estilos de componentes & Entender \\
\hline Decompor um vetor em componentes & Analisar \\
\hline Descreva o que acontece com um vetor quando multiplicado por um escalar & Recordar \\
\hline Organize vetores graficamente para representar adição ou subtração de vetores & Aplicar \\
\hline
\end{tabular}

\section{Resultados e discussões na análise do REA}

Muitos pesquisadores da área da educação se utilizam da taxonomia de Bloom para construir e aplicar suas atividades e testes em suas aulas. Conforme aponta Crowe, Dirks, \& Wenderoth (2008) o alinhamento das atividades e os testes com os resultados da aprendizagem é fundamental para o desenho eficaz de um curso. A pesquisa não teve como objetivo apontar se os professores estão utilizando ou colocando os objetivos de aprendizagem em suas atividades de forma correta, mas de entender que níveis de objetivos de aprendizagem os professores estão se apropriando ou explorando ao usar os OE como base para a construção das suas atividades. Assim a real importância de termos observado o uso dos objetivos de aprendizagem na pesquisa corrobora com Pozo 
\& Gómez Crespo (2009) que esses objetivos são meios necessários para que os estudantes atinjam certas capacidades e formas de pensamento.

Para discutir sobre uso dos objetivos de aprendizagem dos OE PhET originais e das atividades dos professores, podemos usar os valores percentuais da Tabela 2, na qual, podemos visualizar os objetivos de aprendizagem agrupados conforme sua característica pedagógica referenciados pelo nível cognitivo da taxonomia de Bloom. Percebemos que os níveis recordar, entender e avaliar totalizaram os percentuais mais expressivos dos objetivos de aprendizagem dos OE PhET originais. Evidenciamos que os níveis aplicar, analisar e criar não estão sendo muito explorados nos objetivos dos recursos. Já nas atividades dos professores tivemos um valor mais expressivo nos níveis recordar e entender, sendo os mais preponderantes os do nível entender.

Tabela 2. Análise dos objetivos de aprendizagem.

\begin{tabular}{|c|r|r|r|r|}
\hline & \multicolumn{2}{|c|}{$\begin{array}{c}\text { Objetivos de aprendizagem } \\
\text { nos OE PhET originais }\end{array}$} & \multicolumn{2}{c|}{$\begin{array}{l}\text { Objetivos de aprendizagem } \\
\text { nas Atividades Professores }\end{array}$} \\
\hline Níveis cognitivos de aprendizagem Analisados & Percentuais & Itens & Percentuais & itens \\
\hline Recordar & $24,5 \%$ & 23 & $27,9 \%$ & 91 \\
\hline Entender & $24,5 \%$ & 23 & $42 \%$ & 137 \\
\hline Aplicar & $13,8 \%$ & 13 & $9,8 \%$ & 32 \\
\hline Analisar & $7,5 \%$ & 7 & $7,4 \%$ & 24 \\
\hline Avaliar & $24,5 \%$ & 23 & $7,7 \%$ & 25 \\
\hline Criar & $5,3 \%$ & 5 & $5,2 \%$ & 17 \\
\hline $\begin{array}{c}\text { Total de objetivos de aprendizagem dos } \\
\text { 20 Objetos Educacionais }\end{array}$ & & 94 & & 326 \\
\hline
\end{tabular}

A fim de analisarmos a relação entre os objetivos de aprendizagem do $\mathrm{OE} \mathrm{PhET}$ originais e das atividades dos professores apresentamos na Figura 2 a média e o desvio padrão da frequência de ocorrência relativa dos objetivos em cada nível cognitivo da taxonomia. Assim, podemos observar que os objetivos de aprendizagens das atividades dos professores, na maioria dos níveis cognitivos da taxonomia, apresentam uma maior representatividade de uso. No entanto, esses os valores de utilização são bastante próximos, com exceção do nível entender e avaliar. A partir disso poderíamos inferir que os professores se utilizam dos objetivos de aprendizagem propostos pelo OE do $\mathrm{PhET}$ originais.

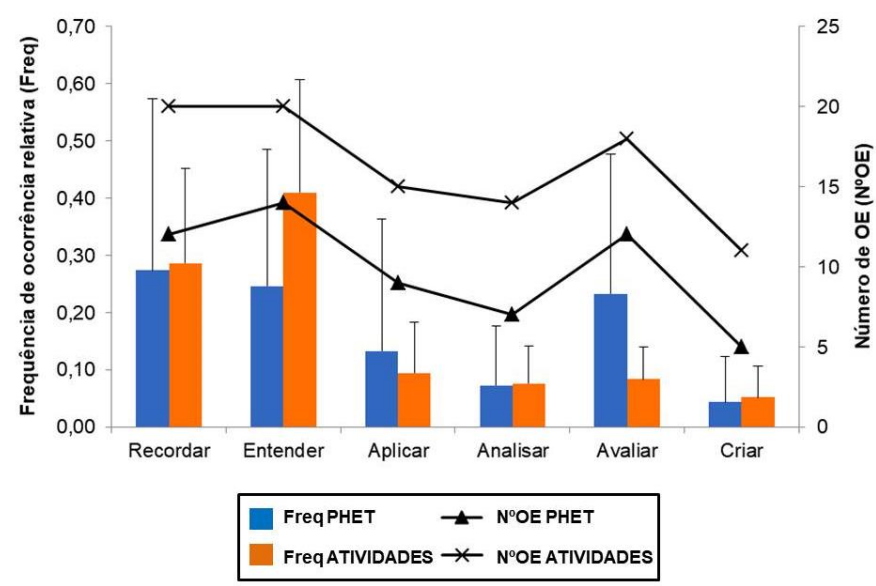

Figura 2. Distribuição dos Objetivos de Aprendizagem nos OE PhET originais e nas Atividades dos Professores. Os valores representam as medias e desvio padrão da frequência de ocorrência relativa (Freq) em cada nível da taxonomia. 
Contudo, quando observamos os objetivos de aprendizagem nos $\mathrm{OE} \mathrm{PhET}$ originais percebemos que apresentam um desvio padrão elevado, o que nos remete que dentro de cada OE há uma diferença de distribuição dos objetivos de cada nível. Assim, existem alguns $\mathrm{OE}$ que tem um número muito elevado de objetivos de aprendizagem, enquanto outros têm poucos ou quase nenhum no referido nível da taxonomia de Bloom. Isso fica evidente quando olhamos o número de $\mathrm{OE}$ em cada nível da taxonomia conforme o eixo secundário da Figura 2, por exemplo, no nível recordar, vemos que apesar dos valores de ocorrência relativa dos OE PhET originais e das atividades serem próximos, o número de $\mathrm{OE}$ que contempla os objetivos de aprendizagem no nível recordar no PhET originais é de $12 \mathrm{OE}$ e das atividades dos professores está presente nos 20 OE. Portanto, percebe-se que a quantidade de objetivos de aprendizagem estão melhor distribuídas ao longo das atividades elaboradas pelos professores, aumentando, com isso, a diversidade de níveis da taxonomia na exploração de cada OE.

Portanto, em cada nível da taxonomia o número de OE explorado por parte dos professores através das atividades foi superior às que foram propostas pelos OE PhET originais. Esse fator que a análise demonstrou sugere que os professores exploraram em suas atividades uma maior diversidade de objetivos de aprendizagem. A partir disso podemos inferir que uma melhor distribuição de objetivos de aprendizagem e níveis da taxonomia podem vir a aumentar a complexidade de exploração dos $\mathrm{OE}$ e a exploração cognitiva de aprendizagem de mais níveis tanto pelos estudantes e como pelos professores.

a)

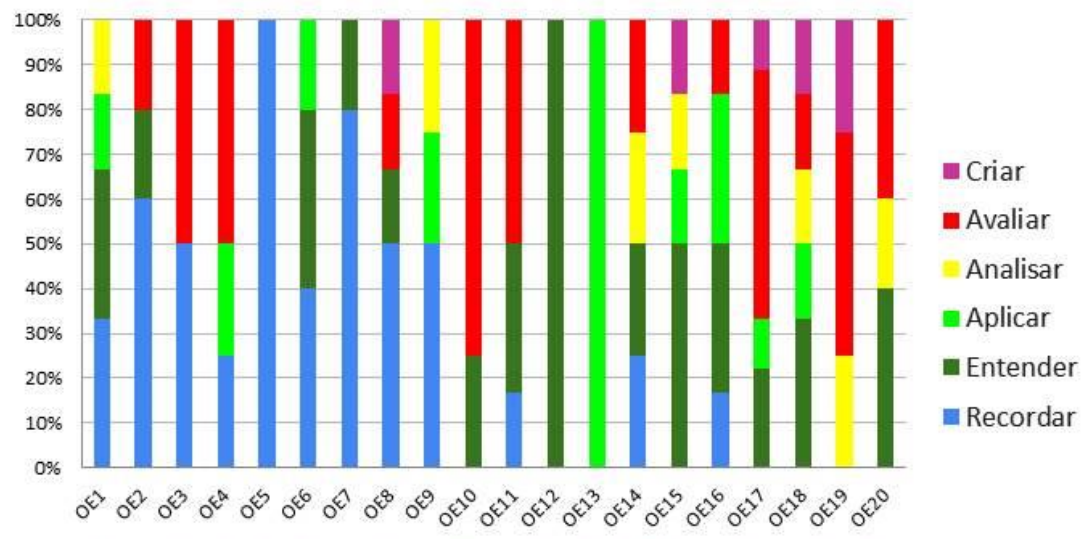

b)

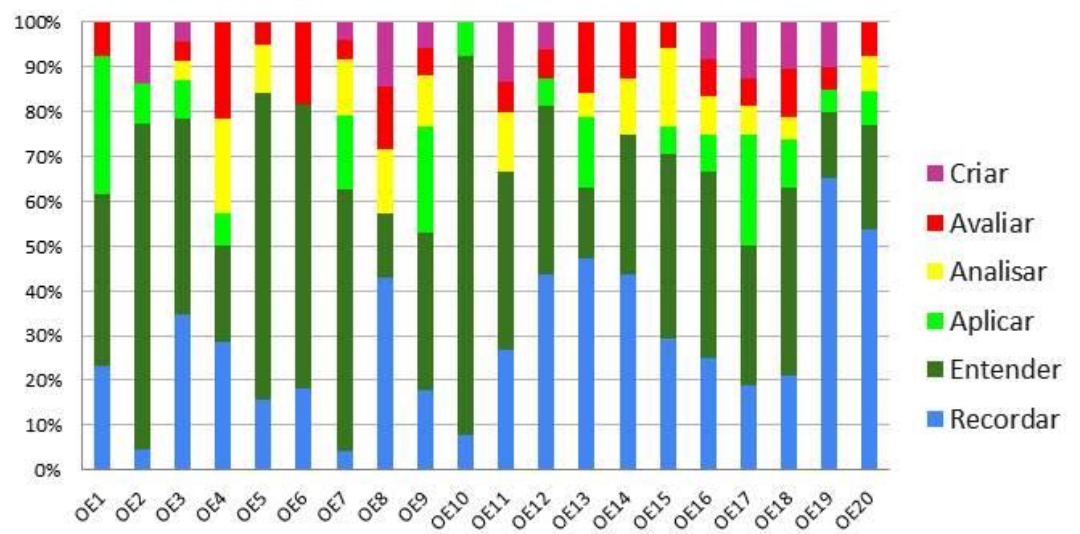

Figura 3. (a) Análise dos objetivos de aprendizagem dos 20 OE PhET originais e (b) Atividades dos professores nos OE 
Com a comparação dos gráficos (a) e (b) da Figura 3 podemos observar a diversidade dos níveis cognitivos de Bloom explorado em cada OE. Nos OE PhET originais, Figura 3 (a), percebe-se que algumas atividades exploram poucos ou apenas um nível da taxonomia na realização dos objetivos de aprendizagem. E em contrapartida na Figura 3 (b) referente às atividades dos professores observamos que os objetivos de aprendizagem contemplam um número mínimo de três níveis, sendo que alguns $\mathrm{OE}$, como os OE 17, 18 e 19, contemplam todos os níveis cognitivos da taxonomia. Desse modo, os professores distribuíram de forma mais diversificada os níveis de aprendizagem ao longo das atividades dos $\mathrm{OE}$, além de explorarem outros níveis cognitivos, os quais não estavam previstos nos objetivos de aprendizagem originalmente proposto pelo $\mathrm{PhET}$.

Durante o processo de análise, podemos observar que algumas atividades propostas pelos professores envolveram outros níveis cognitivos, passando do uso dos níveis básicos do recordar e entender para os níveis mais complexos como avaliar e criar. Segundo Thompson et al (2008) em suas pesquisas foram observadas discrepâncias significativas entre as classificações sugeridas por diferentes professores para uma mesma questão de atividades educacionais, evidenciando que um entendimento sobre a aplicação da taxonomia pode sofrer mudanças de classificação dentro de uma mesma atividade. Isso significa, que a classificação pode ser atualizada ou revisitada, ou mesmo que a atividade pode abranger outros níveis que antes não estavam previstos, com isso, os dados gerados pela análise podem trazer outras possibilidade de uso para os objetivos de aprendizagem dos $\mathrm{OE} \mathrm{PhET}$ que não tenham sido prevista originalmente pelo recurso.

Podemos relatar também, que os professores tiveram uma preocupação em sempre utilizar objetivos de aprendizagens no nível recordar e entender nas atividades em cada OE. Essa preocupação ressalta a importância dos estudantes compreenderem o conteúdo para explorar outros níveis de utilização do $\mathrm{OE}$, mesmo fazendo exercícios de atividades mais complexas como avaliar e criar. Para Pozo \& Gómez Crespo (2009) o uso de bases conceituais é muito importante para a exploração de ações mais complexas no processo de aprendizagem, pois para analisar e avaliar um conteúdo é preciso ter compreendido seus conceitos mais básicos.

Os dados da pesquisa, em relação aos objetivos de aprendizagem, demonstraram que os professores utilizam diversificadas possibilidades de exploração de uso dos OE, vislumbrando atividades de níveis cognitivos mais altos, muitas das quais não estavam previstas originalmente no momento de concepção pelo PhET. Conforme Ávila et al. (2016), a contribuição de professores pode ajudar também na definição de um conjunto de diretrizes e padrões apoiando a nova geração de conhecimento e consequentemente na melhoria dos REA.

Em suma, temos encontrado nos estudos desafios no design de REAs, os quais identificam que a falta de colaboração e envolvimento de professores, estudantes e designers impacta na melhoria do REA, e que considerar o envolvimento dessa partes, resulta em REAs mais significativos e que fazem mais sentido aos usuários [Santana \& Silveira 2017]. A clareza na elaboração, estruturação e construção de um REA pode levar a uma melhor garantia de uso do recurso e potencializar o ensino e aprendizagem. Por outro lado, a participação ativa dos professores na co-criação de um REA contribui 
IX Congresso Brasileiro de Informática na Educação (CBIE 2020)

Anais do XXXI Simpósio Brasileiro de Informática na Educação (SBIE 2020)

para melhoria e desenvolvimento do próprio REA e na construção de outros novos [Ávila, et al. 2016].

\section{Conclusão}

Neste estudo, aplicamos os níveis do domínio cognitivo da Taxonomia Digital de Bloom como uma estrutura teórica para classificar/distinguir os objetivos de aprendizagem das atividades dos professores que estão elencadas com os OE do PhET originais. O estudo possibilitou a visualização de como os professores estão se apropriando dos OE para a elaboração de atividades. A análise conclui que os professores aplicam de modo mais diversificado os objetivos de aprendizagem na construção de suas atividades, e exploram de modo mais harmônico os seis níveis da taxonomia de Bloom. Além de priorizar o uso dos níveis de recordar e entender como objetivos de aprendizagem base para o aprendizado.

Portanto, o PhET poderia apoiar os professores na criação de novas atividades com a utilização de objetivos de aprendizagem como um requisito para $\mathrm{o}$ compartilhamento no repositório contribuindo para a base de dados. Além disso, o PHET poderia explorar esses objetivos postados pelos professores para atualizar os próprios $\mathrm{OE}$ disponibilizados, enriquecendo assim as possibilidades dos recursos. Essas informações poderiam indicar níveis cognitivos que cada estudante trabalharia ao realizar as atividades dos OE. Com isso, a disponibilização de níveis cognitivos em cada atividade poderia auxiliar os estudantes na exploração dos OE permitindo a progressão de níveis mais simples aos mais complexos dos conteúdos dos recursos, maximizando as possibilidades de aprendizagem.

\section{Referencias}

Anderson, L. W., Krathwohl, D. R., and Bloom, B. S. (2001). A Taxonomy for Learning, Teaching, and Assessing a Revision of Bloom's Taxonomy of Educational Objectives, New York, NY: Longman.

Avila, Cecilia et al. Co-criação e avaliação de recursos educacionais abertos inclusivos e acessíveis: Um mapeamento em direção ao paquímetro do IMS. IEEE Revista Iberoamericana de Tecnologias da Aprendizagem, v. 11, n. 3, p. 167-176, 2016.

Bloom, B. S. Some major problems in educational measurement. Journal or Educational Research, v. 38, n. 1, p. 139-142, 1944. BLOOM, B. S. et al. Taxonomy of educational objectives. New York: David Mckay, 1956. 262 p. (v. 1)

Bloom, B. S. Innocence in education. The School Review, v. 80, n. 3, p. 333-352, 1972.

Clark, D. Learning domains of Bloom's taxonomy: the three types of learning. Disponível em: Acesso em: 15 dezembro 2016.

Churches, Andrew. "Taxonomía de Bloom para a era digital." Eduteka. Recuperado 11 (2009). Disponivel em: http://eduteka.icesi.edu.co/articulos/TaxonomiaBloomDigital Acesso em : 20-Jun-2019.

Clements, Kati; Pawlowski, Jan; Manouselis, Nikos. Revisão da literatura sobre repositórios de recursos educacionais abertos - Rumo a uma estrutura abrangente de abordagens de qualidade. Computadores no comportamento humano. v. 51, p. 1098-1106, 2015 . 
Crowe, Alison; Dirks, Clarissa; Wenderoth, Mary Pat. Biologia em flor: implementando a taxonomia de Bloom para aprimorar o aprendizado dos alunos em biologia. CBE Educação em Ciências da Vida , v. 7, n. 4, p. 368-381, 2008.

Diana, Juliana Bordinhão et al. O uso dos repositórios na visão do Designer Instrucional. Revista Brasileira de Informática na Educação, v. 23, n. 01, p. 173, 2015. Disponivel: https://br-ie.org/pub/index.php/rbie/article/viewFile/2836/2857 . Acesso em: 20-Jan-2020.

Dorodchi, Mohsen; Dehbozorgi, Nasrin; Frevert, Tonya K. " I wish I could rank my exam's challenge level!": An algorithm of Bloom's taxonomy in teaching CS1. In: 2017 IEEE Frontiers in Education Conference (FIE). IEEE, 2017. p. 1-5.

Gil, Antonio Carlos. Didática do ensino superior / Antonio Carlos Gil. 1 ed. 2. reimpr. São Paulo: Atlas, 2007.

Guskey, T. R. Benjamin S. Bloom's contributions to curriculum, instruction, and school learning. In: Annual Meeting of the American Educational Research Association, 2001, Seattle. Proceedings... Seattle: AERA

Lomena, M. Benjamin Bloom. Disponível em: http:// www.everything2.com/ index.pl?node_id=143987 Acesso em: 15 dezembro 2016.

Mattar, João. Web 2.0 e redes sociais na educação. João Mattar - São Paulo : Artesanato Educacional, 2013.

Pozo, Juan Ignacio; Crespo, Miguel Angel Gómez. A aprendizagem e o ensino de ciências. Do conhecimento cotidiano ao conhecimento científico, v. 5, 2009.

Santana, Edie, and Ismar Frango Silveira. "Uma Revisão Sistemática Aplicada ao estudo de Recursos Educacionais Abertos Multiculturais." Brazilian Symposium on Computers in Education (Simpósio Brasileiro de Informática na Educação-SBIE). Vol. 28. No. 1. 2017.

School of Education. Bloom's Taxonomy: cognitive domain. Disponível em: http://www.olemiss.edu/depts/ educ_school2/docs/stai_manual/manual8.html. Acesso em: 15 dezembro 2016.

Thompson, Erool et al. "Bloom's taxonomy for CS assessment", In: X Australasian Computing Education Conference - ACE, Australian Computer Society, 2008, p. 155-161.

UNESCO/FRA. Diretrizes para recursos educacionais abertos (REA): no ensino superior. place de Fontenoy, 75352 Paris 07 SP, França. (2015) Disponível em: https://unesdoc.unesco.org/ark:/48223/pf0000232852

Wiley, David A. The instructional use of learning objects. Vol. 1. Bloomington, IN: Agency for instructional technology, 2002. 